\title{
A NOTE ON THEORIES OF LEARNING
}

\author{
JOSEPH PETERSON \\ Peabody College
}

\section{Discussion}

A theory to have scientific value must fulfill at least two conditions which we may state as follows: First, it must picture to us what probably goes on in certain of the gaps between perceived successive processes that cannot be kept under direct continuous observation; that is, where observation is impossible, it must answer plausibly the question How? or What goes on? But it must, in the second place, give this answer in such terms or in such a manner that the hypothecated connection is susceptible of experimental test. If either of these conditions is not fulfilled the theory is not helpful to scientific progress, and in such a case it makes little difference whether or not it is true, if, indeed, truth or falsity can be ascribed to it at all. Often the statement of what goes on under certain conditions must be made in very general and indefinite terms, because of the lack of relevant knowledge; but the theory may still be valuable if it is susceptible of experimental verification and opens the way for more definite supplementary hypotheses as knowledge in the field increases.

The trouble with many of our theories of learning-which are designed to supply knowledge regarding the organic processes going on between the stimuli of certain situations confronted by the individual and the modifications in his responses effected successively by them-is that they have no scientific value, according to the above criteria. It makes no difference whether or not they are true from some standpoints. Such theories we can continue to incorporate in textbooks, as we are doing, and we may require successive generations to learn and pass examinations on them, only to teach in turn to other "learners"; but we get no real returns either in increased knowledge of psychology or in ability to control human nature. It has been encouraging of late to see an increased activity among psychologists toward answering the question, How are errors eliminated in learning so that the fittest acts, of all those brought out by the situations, survive in the form of definitely integrated responses known as habits? 
This is probably one of the most important problems before our science. It seems to me that we understand fairly well how varied responses arise when behavior is impeded or obstructed, but we cannot answer the question just stated. We know, moreover, that when an animal is hungry or under the pressure of some painful stimulus or of some inner "drive," or instinctive determining tendency, it will persist in responses toward rather definite ends, whether consciously anticipated by it or not; but we do not know why it does not in successive trials under similar circumstances continue to repeat random, trial and error, responses characteristic of the first trial. That seems to be the real problem of learning to-day.

A recent experiment reported by Kuo, ${ }^{1}$ from the University of California, divides unsuccessful acts into two classes, merely excessive acts and ill-adaptive acts. The latter are those which check or disrupt activity making toward consummatory reaction, or which bring physical punishment, while the former class are those which merely delay the consummatory act. Kuo experimented with rats in a simple multiple choice apparatus with four compartments, of which one "led the animal to the food box by a short path; another, by a longer path; another confined the animal for a period of time; and a fourth provided an electric shock punishment." Entrance to the second of these compartments was assumed to illustrate excessive acts, while entrance to the last two constituted ill-adaptive acts. It is further assumed "that the electric shock was more ill-adaptive than confinement." Hunger was the primary motive used, and five trials were given each animal daily. Punishment by confinement lasted twenty seconds, and that by electric shock was severe enough to "make the animal squeal every time and immediately jump back from the compartment."

Results show clearly that electric shock is most effective in the elimination of unsuccessful movements, confinement coming next. The long path compartment was eliminated very slowly by all but two rats. "And in many cases the shifting from the long path compartment to the short path compartment came about suddenly and in spite of frequency." Two of the rats eliminated the short path early in the experiment and continued to follow the long one. It is concluded "that frequency and recency as such have very little to do with the matter of elimination in this experiment."

1 Kuo, Z. Y. The nature of Unsuccessful Acts and Their Order of Elimination in Animal Learning. J. of Conipar. Psychol., I922, 2, I-27. 
Various theories of selection and elimination in learning are reviewed and criticized-(1) the pleasure-pain theory, (2) the confirmation and inhibition theory (Hobhouse); (3) the congruity theory (Holmes), (4) the completeness of response theory (Peterson), (5) the intensity theory (Carr), (6) the frequency-recency theory (Watson), and (7) the drive or motor-set theory (Woodworth, Perry, Tolman). The names given in parentheses are those used by the author, who makes little attempt to see similarities and to trace historical developments of the theories or to analyze them. All but the last named of the theories are dismissed as being only descriptive of the facts and not explanatory, while the last is accepted in toto apparently as explanatory, the author overlooking the fact that this theory fails to show the neural mechanism of learning or to suggest why an act is eliminated and not repeated in successive trials. The question of learning is not essentially one of how the animal persists in the activity till it reaches the consummatory act, this being a different problem though somewhat closely related to learning; the real question concerns the mechanism of elimination. This fact is clearly evident in the theories rejected by Kuo as "merely descriptive." It is important to emphasize this point in order that experiments may be directed at the real crux of the problem. It is probably admitted by all investigators now that the inward "drives" to behavior, of which the consummatory reactions are an expression, are not faculties with arbitrary powers but metabolic changes, internal secretions, interstimulation of various part processes in the organism, etc. These factors serve to make the organism react selectively to certain of the numerous exteroceptive stimuli, but how do the erroneous responses-errors from the standpoint of the consummatory act-mecome suppressed and eliminated? It is the how of which we are yet so ignorant, and ignoring it is no solution of the problem. The pleasure-pain theory is hardly stated in scientific terms according to the criteria mentioned in a foregoing paragraph. The same thing is true of the "law of effect," so frequently utilized by some writers; it does not get at the how. The inhibition theory assumes what is to be explained. The intensity theory needs more clarification, and it probably reduces, so far as intensity of response is concerned, to some sort of consistency or congruity conception. The frequency-recency theory is susceptible of experimental investigation of a definite quantitative nature, and in the light of the accumulating evidence it is becoming apparent that frequency-recency factors are in themselves but fixing, not selective, agents in learn- 
ing; that they tend to fix wrong as well as right responses. Several writers have seen this clearly and various drainage theories have been attempted, but the chief difficulty has been in most cases that the theories have not been amenable to experimental test.

As the sponsor to one of the theories rejected by Kuo as descriptive, I wish to point out that this author has apparently overlooked the two experimental studies supporting the completeness of response theory. ${ }^{2}$ The second of these articles analyzes quantitatively the merits of the frequency-recency theories of learning. My own results agree entirely with those of Kuo, but this author seems to miss the nucleus of the difficult problem of learning. The congruity theory seems to be going in the right direction, and I acknowledge much help from its early statement both by $\mathrm{J}$. M. Baldwin and by Holmes; it places the emphasis on the relative consistency of the several impulses to action. This is what I have aimed at in the completeness of response theory, suggesting that the advantage lies with the impulses which give the individual - or the dominant determining tendencies, if this is preferred-the completest expression. It seems to me, however, that to account for the inhibition of the "ill-adaptive acts" we must assume some sort of overlapping of the several stimulus effects so that they can operate simultaneously; and this view certainly has much support from observation-both objective and introspective- of the higher deliberative acts. There is nothing in the motor-set theory accepted by Kuo that is inconsistent with this conception or new to it, but it does not go far enough to meet the real issue. I find much that is good in the motor-set conception of behavior, and accept all of it so far as I know, but cannot regard it as a theory of the selection phenomena in learning, though it may explain selection in response.

2 Petresson, J. The Effect of Length of Blind Alleys on Maze Learning: An Experiment on Twenty-Four White Rats. Behav. Monog., 1917, 3, No. 4; and Frequency and Recency Factors in Maze Learning by White Rats. J. of Animal Behav., 19r7, 7, 338-364. A study on "Learning When Frequency and Recency Factors are Negative" will appear in the $J$. of Exper. Psychol. In this forthcoming article frequency and recency of response are distinguished from frequency and recency of stimulation. 